\title{
Upper and Lower Limb Motor Function Correlates with Ipsilesional Corticospinal Tract and Red Nucleus Structural Integrity in Chronic Stroke: A Cross-Sectional, ROI-Based MRI Study
}

\author{
Denise M. Peters $\left(\mathbb{D},{ }^{1}\right.$ Julius Fridriksson $\mathbb{D}^{2},{ }^{2}$ Jessica D. Richardson ${ }^{D},{ }^{3}$ Jill C. Stewart $\left(\mathbb{D},{ }^{4}\right.$ \\ Chris Rorden $\left(1,{ }^{5}\right.$ Leonardo Bonilha $₫{ }^{6},{ }^{6}$ Addie Middleton $\left(\mathbb{1},{ }^{7}\right.$ and Stacy L. Fritz $\oplus^{4}$ \\ ${ }^{1}$ Department of Rehabilitation and Movement Science, University of Vermont, 106 Carrigan Dr., Burlington, VT, USA \\ ${ }^{2}$ Department of Communication Sciences and Disorders, University of South Carolina, 915 Greene St., Columbia, SC, USA \\ ${ }^{3}$ Department of Speech and Hearing Sciences, University of New Mexico, 1700 Lomas Blvd., Albuquerque, NM, USA \\ ${ }^{4}$ Department of Exercise Science, Physical Therapy Program, University of South Carolina, 921 Assembly St., Columbia, SC, USA \\ ${ }^{5}$ Department of Psychology, University of South Carolina, 1512 Pendleton St., Columbia, SC, USA \\ ${ }^{6}$ Department of Neurology, Medical University of South Carolina, 96 Jonathan Lucas St., Charleston, SC, USA \\ ${ }^{7}$ New England Geriatric Research Education and Clinical Center, VA Boston Healthcare System, 150 South Huntington Ave., \\ Jamaica Plain, MA, USA
}

Correspondence should be addressed to Denise M. Peters; denise.peters@med.uvm.edu

Received 9 August 2021; Accepted 27 October 2021; Published 11 November 2021

Academic Editor: Nicola Tambasco

Copyright (c) 2021 Denise M. Peters et al. This is an open access article distributed under the Creative Commons Attribution License, which permits unrestricted use, distribution, and reproduction in any medium, provided the original work is properly cited.

\begin{abstract}
Background. Structural integrity of the ipsilesional corticospinal tract (CST) is important for upper limb motor recovery after stroke. However, additional neuromechanisms associated with motor function poststroke are less well understood, especially regarding the lower limb. Objective. To investigate the neural basis of upper/lower limb motor deficits poststroke by correlating measures of motor function with diffusion tensor imaging-derived indices of white matter integrity (fractional anisotropy (FA), mean diffusivity (MD)) in primary and secondary motor tracts/structures. Methods. Forty-three individuals with chronic stroke (time poststroke, 64.4 \pm 58.8 months) underwent a comprehensive motor assessment and MRI scanning. Correlation and multiple regression analyses were performed to examine relationships between FA/MD in a priori motor tracts/structures and motor function. Results. FA in the ipsilesional CST and red nucleus (RN) was positively correlated with motor function of both the affected upper and lower limb $(r=0.36-0.55, p \leq 0.01)$, while only ipsilesional RN FA was associated with gait speed $(r=0.50)$. Ipsilesional CST FA explained $37.3 \%$ of the variance in grip strength $(p<0.001)$ and $31.5 \%$ of the variance in Arm Motricity Index $(p=0.004)$. Measures of MD were not predictors of motor performance. Conclusions. Microstructural integrity of the ipsilesional CST is associated with both upper and lower limb motor function poststroke, but appears less important for gait speed. Integrity of the ipsilesional $\mathrm{RN}$ was also associated with motor performance, suggesting increased contributions from secondary motor areas may play a role in supporting chronic motor function and could become a target for interventions.
\end{abstract}

\section{Introduction}

Motor weakness is one of the most disabling consequences of stroke, often leading to difficulties in activities of daily living, gait, and decreased activity levels [1]. Recovery of motor func- tion varies considerably, as patients with similar lesions on structural scans can exhibit different motor impairments and/or responses to treatments. Motor recovery depends on adaptive processes in both the affected and unaffected hemisphere, although the exact neural mechanisms remain unclear $[2,3]$. 
Diffusion tensor imaging (DTI) allows for the examination of the integrity and orientation of white matter in the brain by estimating the magnitude and directionality of water diffusion [4]. Two of the most common DTI-derived metrics are mean diffusivity (MD), which represents the overall magnitude of water diffusion, and fractional anisotropy (FA), which reflects the degree of diffusion directionality [5]. Loss of microstructural integrity of white matter tracts (e.g., local tissue damage within the primary lesion, anterograde, and/or retrograde axonal degeneration) is typically reflected by an increase in MD (representing increased water diffusion in the extracellular space) and/or a reduction in FA (representing decreased anisotropic diffusion) [5]. Axonal properties such as density, myelination, diameter, and orientation contribute to overall FA values [6].

Several studies using DTI techniques have demonstrated a correlation between upper limb motor dysfunction and decreased integrity of white matter tracts in both acute [7, 8] and chronic [9-11] stroke. Reduced structural damage to the ipsilesional corticospinal tract (CST) is associated with better motor outcome after stroke $[9,12]$, yet a significant amount of variance remains unexplained. While the CST is the main motor pathway for voluntary movements of the hand, secondary motor pathways and brain regions also play a role in upper limb motor function and recovery. Microstructural changes in transcallosal fibers (i.e., higher FA values) have also been associated with better motor function poststroke $[9,11]$. Additionally, neuroplastic changes have been shown to occur in the red nucleus (RN) of the affected $[13,14]$ and unaffected hemisphere [14]. The RN (located in the rostral midbrain) is the origin of the rubrospinal tract (RST) [15]. The RST and CST are functionally related with their fibers terminating in close proximity in the spinal cord, suggesting the RN may have some potential to compensate for CST injury following stroke. Studies have suggested that anisotropy within deep nuclear structures (such as the $\mathrm{RN}$ ) may be related to the axon bundles traveling within them [16], and that increased FA values in such nuclei could indicate remodeling and neuroplastic changes after stroke [13, 17].

Motor recovery of the affected upper limb has been highly studied whereas less is known about the neuromechanisms involved with lower limb function after stroke. While the CST is necessary for fine movements of the hands $[18,19]$, locomotion and motor function of the legs is less dependent on the CST [20,21]. Greater structural damage to the CST has been associated with decreased knee extensor strength [22], decreased dorsiflexion and hip flexion movement [23], and increased walking impairment $[23,24]$ in chronic stroke. On the other hand, locomotor ability is present in some stroke survivors despite complete lateral CST injury [20, 21]. In older adults, decreased white matter integrity in the internal capsule and corpus callosum has been associated with gait impairment [25], yet these relationships need further exploration in individuals with stroke.

Overall, these finding suggest that while the integrity of descending neural pathways from the ipsilesional motor system is important for predicting chronic motor function after stroke, contributions from secondary motor areas and tracts may also play a role. Most studies to date have had small sample sizes $(n<20)$, used outcome measures that are not clinically feasible or do not capture different International Classification of Functioning, Disability, and Health (ICF) domains, or focused solely on the CST. Few studies have included measures of subcortical structures or examined chronic white matter integrity and lower limb motor function or gait [24]. We previously reported that cortical disconnection of the ipsilesional primary motor cortex is associated with both gait speed and upper limb motor deficits in chronic stroke [26]. Scalar diffusion parameters such as $\mathrm{FA}$ and $\mathrm{MD}$, however, are more efficient to obtain (in terms of acquisition and postprocessing) than connectivity metrics, making their clinical utility more appealing. Therefore, the aim of the present study was to investigate the neural basis of both upper and lower limb motor deficits in a larger sample of individuals with stroke by correlating measures of motor function with DTI-derived indices of white matter integrity (FA and MD) in primary and secondary motor tracts/regions. We hypothesized that (1) poor upper and lower limb motor function would be correlated with reduced white matter integrity (i.e., lower FA and/or higher MD values) in the ipsilesional CST and corpus callosum, and (2) microstructural changes (as indicated by higher FA values) in secondary motor control regions would be positively associated with upper and lower limb motor performance.

\section{Methods}

2.1. Participants. The same cohort of individuals with chronic stroke involved in our previous connectivity analysis [26] was included in the current analysis (see prior publication for participant demographic, behavioral, and lesion overlay data; https://pericles.pericles-prod.literatumonline .com/doi/epdf/10.1002/hbm.23829). Participants were recruited from January 2013 to June 2014. Eligibility criteria included single left hemispheric stroke $\geq 6$ months (with no maximal time post-stroke cut-off), able to follow simple instructions, and able to independently walk 8 meters with or without an assistive device. Potential participants were excluded if they had contraindications to MRI, clinically reported history of dementia or other neurological condition (e.g., Parkinson's Disease), or extensive visual problems. All participants provided written informed consent approved by the Institutional Review Board at the University of South Carolina.

2.2. Motor Assessment. All participants underwent a comprehensive behavioral assessment of upper and lower limb motor function and gait at our university research lab. Testing was performed by a physical therapist and included the Box and Block Test (BBT) [27] to assess gross manual dexterity of the hand, grip strength [28], Motricity Index (MI) [29] to examine motor function/strength of the upper and lower limbs, and gait speed [30]. Participants used their typical assistive device for the gait speed assessment. 
2.3. MRI Acquisition. All participants underwent scanning using a $3 \mathrm{~T}$ Siemens Trio system with a 12-element head coil at the McCausland Center for Brain Imaging (Columbia, SC) within two days of behavioral testing. High-resolution $3 \mathrm{D} \mathrm{T}_{1}$-MRI scans (repetition time $(\mathrm{TR})=2250 \mathrm{~ms}$, inversion time (TI) $925 \mathrm{~ms}$, echo time $(\mathrm{TE})=4.15 \mathrm{~ms}$, flip angle $=9^{\circ}$, field of view $(\mathrm{FOV})=256 \mathrm{~mm}$, and voxel size $=1.0 \times 1.0 \times$ $1.0 \mathrm{~mm})$ and $3 \mathrm{D} \mathrm{T}_{2}$-MRI scans $(\mathrm{TR}=3200 \mathrm{~ms}, \mathrm{TE}=212$ $\mathrm{ms}$, variable flip angle, $\mathrm{FOV}=256 \mathrm{~mm}$, and voxel size $=1.0$ $\times 1.0 \times 1.0 \mathrm{~mm}$ ) were acquired for determination of lesion size and location. Diffusion imaging was performed with a single-shot gradient echo planar imaging (EPI) monopolar Stejskal-Tanner sequence using the following parameters: $\mathrm{TR}=4987 \mathrm{~ms}, \quad \mathrm{TE}=79.2 \mathrm{~ms}$, flip angle $=90^{\circ}, \quad \mathrm{FOV}=207$ $\mathrm{mm}$, voxel size $=2.3 \times 2.3 \times 2.3 \mathrm{~mm}$, slice thickness $=2.3$ $\mathrm{mm}, 36$ volumes with noncollinear diffusion directions at a $b$ value of $1000 \mathrm{~s} / \mathrm{mm}^{2}$ as well as 5 volumes with a $b$ value of 0 , and number of slices $=50$. Slices were acquired with full Fourier and in-plane parallel acceleration $($ GRAPPA $=2$ ). This diffusion sequence was acquired twice, with the second series reversing the phase-encoding direction.

2.4. Image Preprocessing. Stroke lesions were manually outlined by a neurologist (Bonilha, who was blinded to motor scores) on the T2 image, which was then coregistered to the T1 image. T1-weighted images were normalized into standard MNI space utilizing enantiomorphic unified segmentation-normalization routines as part of the software Statistical Parametric Mapping (SPM) 12 [31], which also applied a lesion-mask cost function [32]. Diffusion images were undistorted using FSL's TOPUP and Eddy tools [33, 34] with excess scalp removed using the FSL BET tool. FSL's dtifit tool was used to compute voxelwise maps of FA and MD. In order to improve registration between T1 and DTI spaces, the scalp-stripped (based on segmentation estimates) T1 image was nonlinearly normalized (using SPM12's "old normalization" function) to match the undistorted FA/MD images.

Region of interest (ROI) analyses were performed using the John Hopkins University (JHU) template overlaid on the voxelwise skeletons of FA and MD. ROIs included bilateral CST, body of the corpus callosum, red nuclei, substantia nigra, thalamus, and superior cerebellar peduncle. We focused our analyses on these ROIs as the CST is the major descending motor pathway and the body of the corpus callosum includes callosal motor fibers connecting cortex motor regions [35]. Furthermore, previous studies have highlighted associations between FA in the body of the corpus callosum and red nucleus and motor performance following stroke $[10,17,36-38]$. We also wanted to elucidate microstructural changes in specific subcortical regions involved in motor control (e.g., substantia nigra, thalamus, and superior cerebellar peduncle) and their relationship with motor function poststroke. The substantia nigra is part of the basal ganglia, a collection of nuclei with extensive brain connections important for postural control and voluntary movement, while the thalamus is a key relay station for sensory-motor neuronal loops involving the cerebellum and basal nuclei [15]. Mean FA and MD values were calculated in each ROI within the JHU atlas. These DTI parameters were chosen due to their commonality in assessing white matter integrity in prior stroke studies and for the different mechanisms they represent (i.e., FA reflects degree of diffusion directionality while MD represents the overall magnitude of water diffusion) [5]. Statistical analyses were performed on ROIs with a lesion load of $<5 \%$ to minimize the influence of necrotic tissue on $\mathrm{FA} / \mathrm{MD}$ values. FA values range from 0 to 1 , with higher values indicating greater structural integrity/directionality.

2.5. Statistical Analyses. Differences in motor scores between affected/unaffected extremities were evaluated using twotailed paired $t$-tests (or Wilcoxon signed-rank tests for nonparametric data). Spearman's correlation analyses were performed to examine the relationship between FA/MD in a priori motor tracts (bilateral CST (at the level of the pons) and body of the corpus callosum) and brain regions (bilateral red nuclei, substantia nigra, thalamus, and superior cerebellar peduncle) and upper/lower limb motor function. Correlations were interpreted as poor $(<0.25)$, fair $(0.25$ to $<0.5)$, moderate $(0.5$ to 0.75$)$, and strong $(>0.75)$ [39].

Regions of interest with a significant bivariate correlation $(p \leq 0.01)$ were entered into regression models to assess the amount of variance in upper and lower limb motor performance explained by the integrity of each ROI. In order to normalize data for regression analyses, participants who scored 0 on BBT were removed as the BBT has a low floor effect [27]. Similarly, participants who scored 99 on the MI were removed due to the high ceiling effect [29]. Separate multiple regressions were performed for each behavioral measure. Age and/or time since stroke were controlled for as covariates if significantly correlated with motor performance. Significance level was set at $p<0.05$, corrected based on the number of behavioral measures assessed (corrected $p \leq 0.01$ ).

An exploratory analysis was performed to investigate if DTI metric-motor behavior relationships differed based on level of motor severity. For upper extremity analyses, BBT scores were used to divide the sample into high (able to move $\geq 80 \%$ of blocks with paretic hand versus nonparetic) and low (able to move $<80 \%$ of blocks) functioning groups. For lower extremity analyses, a gait speed of < 0.8 meters/sec was used as the cut-off for high/low functioning groups which is the cut-off for community ambulator classification [40].

\section{Results}

3.1. Participants. Forty-three individuals with chronic stroke (mean age $59.7 \pm 11.2$ years; time post-stroke $64.4 \pm 58.8$ months; 38 persons right-hand dominant) participated in this study. Power analyses revealed a sample size of 37 would be sensitive (at $80 \%$ power, alpha of 0.05 ) to detect a correlation of 0.4 between upper limb function and ipsilesional CST FA using a bivariate correlation (one-tailed test). All participants exhibited a cortical/subcortical lesion in the left hemisphere, broadly distributed within the territory of the 
middle cerebral artery. Locations of maximal lesion overlap were the left extranuclear and subgyral areas.

3.2. Motor Performance. Motor performance of the affected extremities was significantly reduced compared to the unaffected extremity. The median BBT score for the affected upper limb was 34.5 [interquartile range (IQR), 7.25-46.5] and for the unaffected limb was 51.0 (IQR, 44.0-56.25) $(p<0.001)$. Average grip strength for the affected hand was $23.46 \pm 15.72 \mathrm{~kg}$ and for the unaffected hand was $34.64 \pm$ $10.51 \mathrm{~kg}(p<0.001)$. The median Motricity Index score for the affected upper and lower limb was 88 (IQR, 56.5-100) and 79 (IQR, 59-100), respectively, and for the unaffected limbs was 100 (IQR, 100-100) $(p<0.001)$. Average gait speed was $0.94 \pm 0.31 \mathrm{~m} / \mathrm{s}$. Participants, on average, exhibited a mild to moderate degree of motor impairment.

For the subgroup of participants for the regression analyses, average BBT score for the affected upper limb was $37.1 \pm 17.4(n=32)$, average grip strength for the affected hand was $22.5 \pm 14.8 \mathrm{~kg}(n=39)$, average Arm and Leg MI score for the affected limb was $60.3 \pm 28.6(n=24)$ and $64.9 \pm 18.8(n=27)$, respectively, and average gait speed was $0.95 \pm 0.31 \mathrm{~m} / \mathrm{s}(n=37)$.

3.3. Lesion Size and Motor Function. Total lesion volume was not significantly correlated (Spearman's rho) with motor function of the affected extremities as measured by BBT $(r=-0.17, p=0.29)$, grip strength $(r=0.10, p=0.54)$, Arm MI $(r=-0.15, p=0.37)$, Leg MI $(r=-0.06, p=0.71)$, and gait speed $(r=0.15, p=0.35)$.

3.4. FA/MD in A Priori ROIs and Motor Function. FA values were significantly reduced in the ipsilesional hemisphere compared to the contralesional hemisphere in a priori ROIs $(p<0.01)$, except in the body of the corpus callosum $(p=0.20)$ and superior cerebellar peduncle $(p=0.06)$ (Figure 1). Correlations between upper/lower limb motor function and mean FA values are presented in Table 1. FA in the ipsilesional CST and RN was significantly correlated with motor function of the affected upper limb across all measures, with correlations ranging from $0.36-0.55$. FA in the ipsilesional CST was significantly correlated with motor function of the affected lower limb (Leg $\mathrm{MI}_{\mathrm{Aff}}, r=0.44$ ), and FA in the ipsilesional RN was correlated with both Leg $\mathrm{MI}_{\text {Aff }}$ score $(r=0.49)$ and gait speed $(r=0.50)$. Additionally, FA in the ipsilesional substantia nigra was significantly correlated with Leg $\mathrm{MI}_{\mathrm{Aff}}$ score $(r=0.37)$. No significant correlations were found between affected upper/lower limb motor function and mean FA values in contralesional ROIs.

A significant MD-behavior correlation was observed between the contralesional superior cerebellar peduncle and grip strength of the affected hand $(r=-0.37, p=0.008$, $n=42)$ and Leg MI $(r=-0.40, p=0.005, n=42)$. Otherwise, no significant MD correlations were found across behavioral measures.

For the exploratory analyses, the low functioning group showed similar trends to the overall analysis with stronger correlations demonstrated between ipsilesional CST FA and all upper limb measures $(r=0.71-0.91, p<0.001, n=$

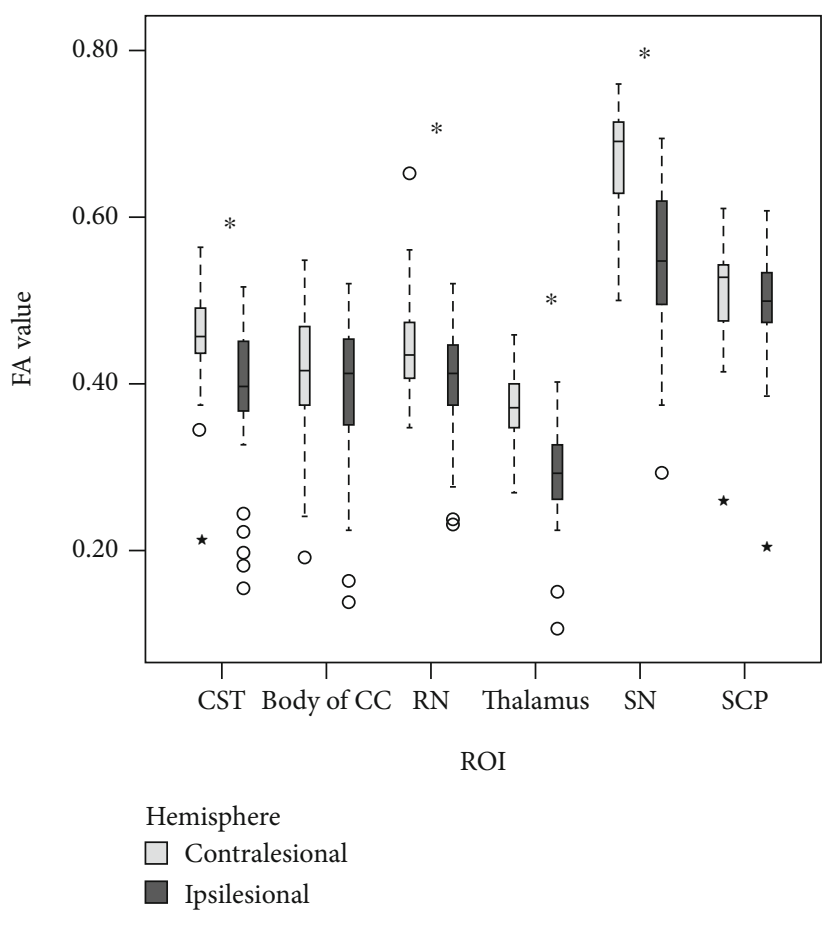

FIgURE 1: Differences in contralesional and ipsilesional structural integrity in a priori regions of interest (ROIs). Reduced fractional anisotropy (FA) values were observed in the ipsilesional hemisphere. The median FA value is represented by the solid black line; circles and stars represent outliers. The range lines indicate the limits of the first and third quartile of the interquartile range. CST: corticospinal tract; CC: corpus callosum; $\mathrm{RN}$ : red nucleus; SN: substantia nigra; SCP: superior cerebellar peduncle. ${ }^{*} p<0.01$ for differences between sides.

18-20 depending on measure) and Leg $\mathrm{MI}_{\mathrm{Aff}}(r=0.72, p=$ $0.001, n=15)$. FA in the ipsilesional $\mathrm{RN}$, however, was only significantly correlated with $\mathrm{BBT}_{\mathrm{Aff}}(r=0.51, p=0.01, n=$ $21)$. Contralesional CST FA was also significantly correlated with all upper limb measures and Leg $\mathrm{MI}_{\text {Aff }}(r=0.61-0.66$, $p \leq 0.005, n=16-21$ depending on measure), while $\mathrm{FA}$ in the contralesional body of the corpus callosum was significantly correlated with gait speed $(r=0.62, p=0.009, n=14$ ). For the high functioning group, only FA in the ipsilesional body of the corpus callosum $(r=-0.72, n=18)$ and thalamus $(r=-0.56, n=18)$ were significantly correlated with grip strength $(p \leq 0.008)$; no other DTI metrics were significantly correlated with motor function in the highfunctioning group.

3.5. Multiple Regression Analyses. Only the two ROIs with the strongest bivariate correlation with motor function were entered into the regression models due to our sample size. After controlling for significant covariates, ipsilesional CST FA was entered first into each model as the CST is the major neural pathway for skilled, discrete voluntary movements [18]. Ipsilesional RN FA was the next predictor entered into the models. The gait speed regression model did not include ipsilesional CST FA, however, as it was not significantly correlated with gait speed. A significant, positive correlation was found between ipsilesional CST and RN FA 
TABLE 1: Nonparametric correlations between motor function and mean FA values in a priori ROIs.

\begin{tabular}{|c|c|c|c|c|c|}
\hline \multirow{2}{*}{ FA in a priori ROIs } & \multicolumn{5}{|c|}{ Behavioral measures } \\
\hline & $\operatorname{BBT}_{\text {Aff }}(n=42) \dagger$ & $\operatorname{Grip}_{\text {Aff }}(n=42) \dagger$ & $\operatorname{Arm~} \mathrm{MI}_{\mathrm{Aff}}(n=40) \dagger$ & Leg $\mathrm{MI}_{\text {Aff }}(n=42) \dagger$ & Gait speed $(n=40) \dagger$ \\
\hline \multicolumn{6}{|l|}{ Ipsilesional } \\
\hline CST & $0.55^{* *}(n=41)$ & $0.38^{*}(n=41)$ & $0.52^{* *}(n=39)$ & $0.44^{*}(n=41)$ & $0.35(n=39)$ \\
\hline Body of CC & $0.05(n=34)$ & $-0.25(n=34)$ & $0.12(n=33)$ & $-0.03(n=34)$ & $-0.27(n=33)$ \\
\hline Red nucleus & $0.45^{* *}$ & $0.36^{*}$ & $0.45^{*}$ & $0.49^{* *}$ & $0.50^{* *}$ \\
\hline Thalamus & $0.33(n=33)$ & $0.11(n=33)$ & $0.39(n=32)$ & $0.14(n=34)$ & $-0.12(n=32)$ \\
\hline Substantia nigra & $0.36^{*}(n=41)$ & $0.19(n=41)$ & $0.36(n=39)$ & $0.37^{*}(n=41)$ & $0.17(n=39)$ \\
\hline SCP & 0.10 & 0.17 & 0.19 & 0.26 & 0.20 \\
\hline \multicolumn{6}{|l|}{ Contralesional } \\
\hline CST & 0.13 & 0.23 & 0.15 & 0.11 & 0.11 \\
\hline Body of CC & 0.11 & -0.05 & 0.10 & 0.01 & -0.04 \\
\hline Red nucleus & 0.01 & -0.08 & 0.06 & 0.08 & 0.22 \\
\hline Thalamus & 0.02 & 0.07 & 0.02 & -0.02 & -0.19 \\
\hline Substantia nigra & -0.25 & -0.24 & -0.26 & -0.25 & -0.15 \\
\hline SCP & 0.09 & 0.26 & 0.18 & 0.30 & 0.36 \\
\hline
\end{tabular}

Values in the table are Spearman's coefficients $(r)$. Abbreviations: ROIs: regions of interest; BBT $_{\mathrm{Aff}}$ : Box and Block Test (affected limb); Grip ${ }_{\mathrm{Aff}}$ grip strength (affected limb); Arm $\mathrm{MI}_{\mathrm{Aff}}$ : Arm Motricity Index score (affected limb); Leg $\mathrm{MI}_{\mathrm{Aff}}$ Leg Motricity Index score (affected limb); CST: corticospinal tract; CC: corpus callosum; SCP: superior cerebellar peduncle. †Overall $n$ value ( $n$ exceptions noted in parentheses, accounting for ROIs with $\geq 5 \%$ lesion volume). ${ }^{* *} p \leq 0.001 ;{ }^{*} p \leq 0.01$.

(Spearman's $r=0.42, p=0.01, n=42$ ), yet since the correlation between these independent variables was fairly low, both variables were retained for analyses (Table 2). Ipsilesional CST FA significantly explained $37.3 \%$ of the variance in grip strength of the affected hand $(F(1,38)=22.00, p<$ $0.001)$ and $31.5 \%$ of the variance in Arm $\mathrm{MI}_{\mathrm{Aff}}$ score $(F(1,23)=10.11, p=0.004)$. Adding ipsilesional RN FA to the models, however, did not significantly explain an additional amount of variance in upper limb motor performance. No other regression analyses revealed significant models (at corrected $p \leq 0.01$ ), but several models approached significance (at $p \leq 0.05$ ). Multiple regression analyses were not conducted for the exploratory analyses due to the small sample sizes within each subgroup.

\section{Discussion}

This study examined relationships between structural integrity of different motor tracts/brain regions and affected upper and lower limb motor function in chronic stroke. Integrity of the ipsilesional CST, RN, thalamus, and substantia nigra was significantly lower compared to homologous regions in the contralesional hemisphere. Variability in the structural integrity of the ipsilesional CST not only correlated with affected upper limb motor function but also with lower limb strength; however, the CST appears to be less important for gait speed. Furthermore, structural integrity of the ipsilesional $\mathrm{RN}$ was associated with upper and lower limb motor performance, including gait speed, suggesting this region may contribute to motor outcome in chronic stroke. This suggestion is supported by findings that rubral branch integrity is associated with upper and lower limb motor impairment in chronic stroke [41, 42], as well as lesion studies in animals and neuroimaging studies in humans correlating the RN with motor function [43]. This study is novel in that it included both cortical and subcortical measures of structural integrity and examined brainbehavior relationships as it relates to both upper and lower limb motor function. Integrity of not only the CST but other brain structures involved in motor control may play a role in subsequent long-term motor outcome in chronic stroke [44-46].

Our findings provide further evidence that preservation of ipsilesional CST integrity is critical for chronic motor function poststroke $[9,12]$. Less is known, however, about the contribution and microstructural changes of the contralesional CST after stroke. Schaechter et al. [47] found that FA in both the ipsilesional and contralesional CST was significantly and positively correlated with motor skill performance of patients' affected hand. Accumulating evidence indicates that white matter remodeling occurs in both ipsilesional and contralesional hemispheres, suggesting that structural remodeling of the contralesional motor system also contributes to motor recovery after stroke [3, 48]. Our results, however, did not find a significant correlation between contralesional CST FA and motor performance of the affected upper/lower limbs. This difference could be attributed to different sample characteristics (e.g., higher degree of motor impairment in previous studies compared to the current study) and/or to differences in measures of motor function.

FA in the ipsilesional $\mathrm{RN}$ was also associated with affected upper and lower limb motor function. Anisotropy within the RN has been postulated to be associated with its afferent and efferent fibers [16], such as the rubrospinal tract (RST). Animal studies have shown that the RST has connections with contralateral spinal motor neurons [49] and have suggested a compensatory role of the RST in motor recovery 
TABLe 2: Multiple regression analyses of tract/region-specific FA and upper and lower limb motor performance.

\begin{tabular}{|c|c|c|c|c|c|c|c|}
\hline Behavioral measure & Predictors & $R^{2}$ & $F$ statistic & $p$ value for $F$ & $\beta_{\mathrm{TSS}}$ & $\beta_{\mathrm{CST}}$ & $\beta_{\mathrm{RN}}$ \\
\hline \multicolumn{8}{|l|}{$\mathrm{BBT}_{\mathrm{Aff}}(n=32)^{\mathrm{a}}$} \\
\hline Model 1 & TSS, FA CST $_{\text {ipsi }}$ & 0.264 & 5.21 & 0.012 & -0.258 & 0.335 & \\
\hline Model 2 & TSS, FA CST $_{\text {ipsi }}$, FA RN ipsi & 0.270 & 3.45 & 0.030 & -0.230 & 0.344 & 0.076 \\
\hline \multicolumn{8}{|l|}{$\operatorname{Grip}_{\text {Aff }}(n=39)^{\mathrm{a}}$} \\
\hline Model 1 & FA CST $_{\text {ipsi }}$ & 0.373 & 22.03 & $\leq 0.001 \dagger$ & & $0.611^{* *}$ & \\
\hline Model 2 & $\mathrm{FA} \mathrm{CST}_{\mathrm{ipsi}}, \mathrm{FA} \mathrm{RN}_{\mathrm{ipsi}}$ & 0.374 & 10.74 & $\leq 0.001 \dagger$ & & $0.597^{* *}$ & 0.027 \\
\hline \multicolumn{8}{|l|}{$\operatorname{Arm~} \mathrm{MI}_{\mathrm{Aff}}(n=24)^{\mathrm{b}}$} \\
\hline Model 1 & FA CST $_{\text {ipsi }}$ & 0.315 & 10.13 & $0.004 \dagger$ & & $-0.561^{* *}$ & \\
\hline Model 2 & $\mathrm{FA} \mathrm{CST}_{\mathrm{ipsi}}, \mathrm{FA} \mathrm{RN}_{\mathrm{ipsi}}$ & 0.337 & 5.34 & 0.013 & & $-0.472^{*}$ & -0.173 \\
\hline \multicolumn{8}{|l|}{ Leg $\mathrm{MI}_{\mathrm{Aff}}(n=27)$} \\
\hline Model 1 & $\mathrm{FA} \mathrm{CST}_{\mathrm{ipsi}}$ & 0.186 & 5.72 & 0.025 & & $0.432^{*}$ & \\
\hline Model 2 & $\mathrm{FA} \mathrm{CST}_{\mathrm{ipsi}}, \mathrm{FA} \mathrm{RN_{ \textrm {ipsi } }}$ & 0.241 & 3.82 & 0.036 & & 0.282 & 0.278 \\
\hline \multicolumn{8}{|l|}{ Gait speed $(n=39)^{\mathrm{c}}$} \\
\hline Model 1 & TSS, FA RN $\mathrm{ipsi}$ & 0.237 & 5.82 & 0.021 & -0.239 & - & $0.365 *$ \\
\hline
\end{tabular}

following CST injury in nonhuman primates [50]. Although the RST is more anatomically prominent in animals compared with humans [51], the RST may undergo neuroplastic changes poststroke that promotes increased functional contribution to motor recovery. Only a few studies, however, have examined neuroplastic changes in the RN following CST injury in humans after stroke. In persons 8-21 days poststroke, Yeo and Jang [13] found higher mean FA in the ipsilesional RN compared to the contralesional RN and healthy controls. Takenobu et al. [8] also found increased FA in the ipsilesional RN compared to the contralesional $\mathrm{RN}$ at 3 months poststroke, with a positive correlation with motor recovery. Rüber et al. [17] found higher FA values in both ipsilesional and contralesional RN in 18 chronic stroke patients compared to healthy controls, with significant positive correlations between red nuclei FA and level of motor function. These results suggest that remodeling and neuroplastic changes occur in the $\mathrm{RN}$ during the early stages of stroke and are still evident in the chronic stage and that these changes may indicate compensation for CST injury and contribute to motor outcome. Our results show that variability in upper and lower limb motor function in chronic stroke is associated with variability in ipsilesional RN FA, although we cannot directly infer a compensatory role of the RST in motor recovery. The human RN receives extensive input from the cerebral cortex and is involved in grasping, motor control, and coordination [43]. The RN may also play an important role in the neonatal brain, with regression in the adult brain linked to the development of upright bipedalism [52]. However, more clarity is needed regarding the function of the human $\mathrm{RN}$ and its role in motor recovery poststroke.

Of the other motor subnetwork a priori ROIs, only FA in the ipsilesional substantia nigra (SN) revealed a significant relationship with chronic motor performance $\left(\mathrm{BBT}_{\mathrm{Aff}}\right.$ and
Leg $\left.\mathrm{MI}_{\mathrm{Aff}}\right)$. The $\mathrm{SN}$ is part of the basal ganglia and is involved in motor control. It receives input from other basal nuclei, the cerebral cortex, and midbrain reticular formation; efferent information passes back to the cerebral cortex, basal ganglia, red nucleus, thalamus, and amygdala [15]. Our results suggest that ipsilesional $\mathrm{SN}$ integrity may also play a role in upper and lower limb motor outcome in chronic stroke.

In contrast to previous studies $[8,10,36]$, we did not find significant correlations between the microstructural integrity of the corpus callosum and motor performance. This difference could be attributed to different sample characteristics (higher versus lower functioning), different motor assessments (e.g., prior studies commonly used the Fugl-Meyer assessment), and/or differences in how the corpus callosum was delineated or whether lesioned voxels were included in the analyses. We examined only the body of the corpus callosum, which contains the commissural fibers connecting bilateral motor cortices, using the JHU ROI atlas and only included ROIs with $<5 \%$ lesion volume in our analyses.

Regression analyses revealed that ipsilesional CST FA was a predictor of grip strength and Arm $\mathrm{MI}_{\text {Aff }}$ score. These results complement other studies that have shown integrity of the ipsilesional CST $[2,36]$ or CST/PLIC FA asymmetry $[9,19]$ is related to upper limb motor performance poststroke. Adding ipsilesional RN FA to the models did not significantly explain more variance in motor performance. In terms of lower limb motor function, neither ipsilesional CST or RN FA were significant predictors. This finding may be a reflection of the complex neural circuitry involved with locomotion. The neural control of upper and lower limb movements is not analogous, as spinal interneurons play a role in the central pattern generation of gait [53] while fine hand movements are primarily under cerebral control. A few studies have suggested that the lateral CST does not 
play a central role in basic locomotor function in primates or humans [20], but rather is involved in gait adaptation [54]. Paretic leg movements and locomotor ability have also been present in some stroke survivors despite complete lateral CST injury [20,21]. Other descending neural pathways such as the reticulospinal, rubrospinal, and vestibulospinal tracts could contribute to locomotor function and recovery after stroke. As stated previously, significant correlations were found between ipsilesional RN FA and lower limb motor performance in our study.

In our exploratory analyses examining brain-behavior relationships based on level of motor severity, the low functioning group exhibited similar trends (with stronger correlations) between ipsilesional CST FA and upper/lower limb motor function, providing further evidence that reduced structural damage to the ipsilesional CST is important for motor recovery in individuals with more severe motor impairment $[9,55,56]$. Ipsilesional RN FA was still correlated with $\mathrm{BBT}_{\mathrm{Aff}}$ but was no longer correlated with grip strength, Motricity Index, or gait speed at the corrected $p$ value of $\leq 0.01$. Additionally, contralesional CST FA correlated with upper limb motor function across all measures. This finding is in line with prior work suggesting that the contralesional CST plays a compensatory role to support paretic upper limb movements in individuals with more severe motor deficits $[47,57,58]$. In contrast, only FA in the ipsilesional body of the corpus callosum and thalamus correlated with paretic hand function in the high functioning group. Recent work has found that motor function in individuals with mild upper limb impairments is correlated with integrity of the corpus callosum but not the CST [59, 60], suggesting that interhemispheric structural connections between motor cortices play a supportive role in chronic motor recovery in this population. Regarding lower limb brain-behavior relationships, bilateral CST FA correlated with affected lower limb strength and FA in the contralesional body of the corpus callosum correlated with gait speed, but only in the low functioning group. Research has demonstrated less lateralization of cortical activity with leg movements compared to hand movements [61], which is not surprising as lower limb movements are mainly bilateral.

Regarding mean diffusivity, significant findings between $\mathrm{MD}$ and motor function were minimal. Only a few studies have reported significant relationships between white matter integrity (as indexed by MD) and sensorimotor function in chronic stroke [62-64], while others have found no relationship $[65,66]$. Furthermore, while MD values can change quite rapidly after stroke, MD has been shown to be pseudonormalized within 96 hours poststroke in animals models $[67,68]$.

This study is one of the first to incorporate a large sample size with left hemisphere necrotic damage when examining the relationship between motor function and microstructural integrity in chronic stroke and may explain some of the differences with previous studies. As there are hemispheric differences in motor structure and activation related to motor attention, action selection, and task complexity $[69,70]$, examining changes in structural integrity following right hemispheric stroke and relationships to motor function is also imperative. Increasing our under- standing of the relationship between structural integrity of multiple motor pathways and motor function poststroke may provide greater insight into mechanisms that support brain plasticity and motor recovery. Such information could improve motor recovery prognosis and assist with targeting therapeutic interventions. For example, researchers and clinicians could design treatment approaches that utilize different motor pathways/brain regions depending on structural integrity metrics of specific motor pathways. Associations between upper limb motor impairment and microstructural integrity of the CST [9], transcallosal tracts [10], and extrapyramidal pathways (rubrospinal and reticulospinal) [42] in chronic stroke highlight the importance of examining multiple motor pathways/brain regions as potential biomarkers for motor outcome and response to treatment. Future studies examining training-induced neuroplastic changes are also warranted as treatment-related white matter changes (i.e., increase in FA pre-posttreatment) have been associated with both speech [71] and upper limb motor gains [72] in chronic stroke.

Our findings need to be interpreted in the context of our study design and the limitations of DTI and FA. The measured diffusion tensor is an average of several tissue compartments with different diffusion profiles within each voxel. Therefore, areas of partial tissue volume (mixture of white matter/gray matter/cerebrospinal fluid) or white matter partial volume (crossing or diverging fibers) will result in low anisotropy [73]. Furthermore, many factors influence FA values (e.g., axonal count/density and fiber organization) and DTI cannot discern which structural element(s) are contributing to observed changes in FA. Additionally, our ROIs were defined by the atlas we used; for example, we assessed only the distal portion of the CST. Other studies have used alternative analysis techniques that incorporate different ROI parameters, utilize a tract-based approach, or evaluate FA at different levels along motor tracts [9, 19, 36, 47]. While each method has its advantages and limitations, the different approaches are able to detect decreased FA values in persons poststroke and identify behavioral correlations [74], and the optimal approach for the investigation of brain-behavior relationships remains unclear. Further, using ROIs from an atlas developed in healthy adults is critically dependent on spatial normalization quality, such that larger lesions may cause more atrophy leading to a larger displacement of a region (though we note that we used state-of-theart normalization methods). Lastly, our sample included a large number of subjects with mild motor impairment; results may not extrapolate to individuals with more severe impairment.

\section{Conclusions}

Our findings demonstrate that microstructural integrity of the ipsilesional CST is correlated with both affected upper and lower limb motor function across different ICF domains in chronic stroke. The CST appears to be less important, however, for gait speed than to the control of upper limb dexterity and strength. Additionally, microstructural integrity of the ipsilesional $\mathrm{RN}$ was fair to moderately associate 
with motor performance of the affected limbs, suggesting that this region may contribute to motor outcome poststroke. Ipsilesional RN FA, however, did not significantly explain an additional amount of variance in upper limb strength beyond that explained by ipsilesional CST FA. Further elucidation of the role that secondary motor pathways which plays in chronic motor function is needed, as well as relationships between structural and functional reorganization. Such insight could help with targeting rehabilitation techniques in an effort to optimize motor recovery following stroke.

\section{Data Availability}

Data are available from the corresponding author upon request.

\section{Conflicts of Interest}

The authors declare that there are no conflicts of interest regarding the publication of this paper.

\section{Acknowledgments}

Research, authorship, and/or publication of this article was supported by the National Institute on Deafness and other Communication Disorders (NIH/NIDCD: DC014664) and the National Institute of General Medical Sciences (NIH/NIGMS: P20GM135007, Vermont Center for Cardiovascular and Brain Health).

\section{References}

[1] P. W. Duncan, L. B. Goldstein, D. Matchar, G. W. Divine, and J. Feussner, "Measurement of motor recovery after stroke. Outcome assessment and sample size requirements," Stroke, vol. 23, no. 8, pp. 1084-1089, 1992.

[2] L. L. Zhu, R. Lindenberg, M. P. Alexander, and G. Schlaug, "Lesion load of the corticospinal tract predicts motor impairment in chronic stroke," Stroke, vol. 41, no. 5, pp. 910-915, 2010.

[3] J. C. Eliassen, E. L. Boespflug, M. Lamy, J. Allendorfer, W. J. Chu, and J. P. Szaflarski, "Brain-mapping techniques for evaluating poststroke recovery and rehabilitation: a review," Topics in Stroke Rehabilitation, vol. 15, no. 5, pp. 427-450, 2008.

[4] P. C. Sundgren, Q. Dong, D. Gomez-Hassan, S. K. Mukherji, P. Maly, and R. Welsh, "Diffusion tensor imaging of the brain: review of clinical applications," Neuroradiology, vol. 46, no. 5, pp. 339-350, 2004.

[5] S. H. Fung, L. Roccatagliata, R. G. Gonzalez, and P. W. Schaefer, "MR diffusion imaging in ischemic stroke," Neuroimaging Clinics of North America, vol. 21, no. 2, pp. 345-377, 2011.

[6] C. Beaulieu, "The biological basis of diffusion anisotropy," in Diffusion MRI: From Quantitative Measurement to In Vivo Neuroanatomy, H. Johansen-Berg and T. Behrens, Eds., Elsevier, London, 2009.

[7] B. Radlinska, S. Ghinani, I. R. Leppert, J. Minuk, G. B. Pike, and A. Thiel, "Diffusion tensor imaging, permanent pyramidal tract damage, and outcome in subcortical stroke," Neurology, vol. 75 , no. 12, pp. 1048-1054, 2010.
[8] Y. Takenobu, T. Hayashi, H. Moriwaki, K. Nagatsuka, H. Naritomi, and H. Fukuyama, "Motor recovery and microstructural change in rubro-spinal tract in subcortical stroke," NeuroImage: Clinical, vol. 4, pp. 201-208, 2014.

[9] R. Lindenberg, V. Renga, L. L. Zhu, F. Betzler, D. Alsop, and G. Schlaug, "Structural integrity of corticospinal motor fibers predicts motor impairment in chronic stroke," Neurology, vol. 74, no. 4, pp. 280-287, 2010.

[10] J. L. Chen and G. Schlaug, "Resting state interhemispheric motor connectivity and white matter integrity correlate with motor impairment in chronic stroke," Frontiers in Neurology, vol. 4, p. 178, 2013.

[11] A. Sterr, P. J. Dean, A. J. Szameitat, A. B. Conforto, and S. Shen, "Corticospinal tract integrity and lesion volume play different roles in chronic hemiparesis and its improvement through motor practice," Neurorehabilitation and Neural Repair, vol. 28, no. 4, pp. 335-343, 2014.

[12] R. Pineiro, S. T. Pendlebury, S. Smith et al., "Relating MRI changes to motor deficit after ischemic stroke by segmentation of functional motor pathways," Stroke, vol. 31, no. 3, pp. 672679,2000

[13] S. S. Yeo and S. H. Jang, "Changes in red nucleus after pyramidal tract injury in patients with cerebral infarct," NeuroRehabilitation, vol. 27, no. 4, pp. 373-377, 2010.

[14] Y. Dong, C. J. Winstein, R. Albistegui-DuBois, and B. H. Dobkin, "Evolution of FMRI activation in the perilesional primary motor cortex and cerebellum with rehabilitation trainingrelated motor gains after stroke: a pilot study," Neurorehabilitation and Neural Repair, vol. 21, no. 5, pp. 412-428, 2007.

[15] A. K. Afifi and R. A. Bergman, Functional Neuroanatomy, McGraw-Hill, New York, NY, 1998.

[16] C. Habas and E. A. Cabanis, "Cortical projection to the human red nucleus: complementary results with probabilistic tractography at 3 T," Neuroradiology, vol. 49, no. 9, pp. 777-784, 2007.

[17] T. Ruber, G. Schlaug, and R. Lindenberg, "Compensatory role of the cortico-rubro-spinal tract in motor recovery after stroke," Neurology, vol. 79, no. 6, pp. 515-522, 2012.

[18] R. A. Davidoff, “The pyramidal tract,” Neurology, vol. 40, no. 2, pp. 332-339, 1990.

[19] C. M. Stinear, P. A. Barber, P. R. Smale, J. P. Coxon, M. K. Fleming, and W. D. Byblow, "Functional potential in chronic stroke patients depends on corticospinal tract integrity," Brain, vol. 130, Part 1, pp. 170-180, 2007.

[20] Y. H. Ahn, S. H. Ahn, H. Kim, J. H. Hong, and S. H. Jang, "Can stroke patients walk after complete lateral corticospinal tract injury of the affected hemisphere?," Neuroreport, vol. 17, no. 10, pp. 987-990, 2006.

[21] S. H. Jang, S. H. You, Y. H. Kwon, M. Hallett, M. Y. Lee, and S. H. Ahn, "Cortical reorganization associated lower extremity motor recovery as evidenced by functional MRI and diffusion tensor tractography in a stroke patient," Restorative Neurology and Neuroscience, vol. 23, no. 5-6, pp. 325-329, 2005.

[22] S. Madhavan, C. Krishnan, A. Jayaraman, W. Z. Rymer, and J. W. Stinear, "Corticospinal tract integrity correlates with knee extensor weakness in chronic stroke survivors," Clinical Neurophysiology, vol. 122, no. 8, pp. 1588-1594, 2011.

[23] J. P. Seo, K. H. Do, G. S. Jung et al., "The difference of gait pattern according to the state of the corticospinal tract in chronic hemiparetic stroke patients," NeuroRehabilitation, vol. 34, no. 2, pp. 259-266, 2014. 
[24] G. Jayaram, C. J. Stagg, P. Esser, U. Kischka, J. Stinear, and H. Johansen-Berg, "Relationships between functional and structural corticospinal tract integrity and walking post stroke," Clinical Neurophysiology, vol. 123, no. 12, pp. 24222428, 2012.

[25] R. A. Bhadelia, L. L. Price, K. L. Tedesco et al., "Diffusion tensor imaging, white matter lesions, the corpus callosum, and gait in the elderly," Stroke, vol. 40, no. 12, pp. 3816-3820, 2009.

[26] D. M. Peters, J. Fridriksson, J. C. Stewart et al., "Cortical disconnection of the ipsilesional primary motor cortex is associated with gait speed and upper extremity motor impairment in chronic left hemispheric stroke," Human Brain Mapping, vol. 39, no. 1, pp. 120-132, 2018.

[27] H. M. Chen, C. C. Chen, I. P. Hsueh, S. L. Huang, and C. L. Hsieh, "Test-retest reproducibility and smallest real difference of 5 hand function tests in patients with stroke," Neurorehabilitation and Neural Repair, vol. 23, no. 5, pp. 435-440, 2009.

[28] D. L. Riddle, S. D. Finucane, J. M. Rothstein, and M. L. Walker, "Intrasession and intersession reliability of hand-held dynamometer measurements taken on brain-damaged patients," Physical Therapy, vol. 69, no. 3, pp. 182-189, 1989.

[29] G. Demeurisse, O. Demol, and E. Robaye, "Motor evaluation in vascular hemiplegia," European Neurology, vol. 19, no. 6, pp. 382-389, 2004.

[30] D. S. Stokic, T. S. Horn, J. M. Ramshur, and J. W. Chow, "Agreement between temporospatial gait parameters of an electronic walkway and a motion capture system in healthy and chronic stroke populations," American Journal of Physical Medicine \& Rehabilitation, vol. 88, no. 6, pp. 437-444, 2009.

[31] C. Rorden, L. Bonilha, J. Fridriksson, B. Bender, and H. O. Karnath, "Age-specific CT and MRI templates for spatial normalization,” NeuroImage, vol. 61, no. 4, pp. 957-965, 2012.

[32] M. Brett, A. P. Leff, C. Rorden, and J. Ashburner, "Spatial normalization of brain images with focal lesions using cost function masking," NeuroImage, vol. 14, no. 2, pp. 486-500, 2001.

[33] J. L. Andersson, S. Skare, and J. Ashburner, "How to correct susceptibility distortions in spin-echo echo-planar images: application to diffusion tensor imaging," NeuroImage, vol. 20, no. 2, pp. 870-888, 2003.

[34] J. L. R. Andersson and S. N. Sotiropoulos, "An integrated approach to correction for off-resonance effects and subject movement in diffusion MR imaging," NeuroImage, vol. 125, pp. 1063-1078, 2016.

[35] M. Wahl, B. Lauterbach-Soon, E. Hattingen et al., "Human motor corpus callosum: topography, somatotopy, and link between microstructure and function," The Journal of Neuroscience, vol. 27, no. 45, pp. 12132-12138, 2007.

[36] R. Lindenberg, L. L. Zhu, T. Ruber, and G. Schlaug, "Predicting functional motor potential in chronic stroke patients using diffusion tensor imaging," Human Brain Mapping, vol. 33, no. 5, pp. 1040-1051, 2012.

[37] H. Kim, H. Lee, K. I. Jung, S. H. Ohn, and W. K. Yoo, "Changes in diffusion metrics of the red nucleus in chronic stroke patients with severe corticospinal tract injury: a preliminary study," Annals of Rehabilitation Medicine, vol. 42, no. 3, pp. 396-405, 2018.

[38] Y. Li, P. Wu, F. Liang, and W. Huang, "The microstructural status of the corpus callosum is associated with the degree of motor function and neurological deficit in stroke patients," PLoS One, vol. 10, no. 4, article e0122615, 2015.
[39] L. G. Portney and M. P. Watkins, "Correlation," in Foundations of Clinical Research: Applications to Practice, p. 494, Prentice Hall, Upper Saddle River, NJ, 2nd edition, 2000.

[40] J. Perry, M. Garrett, J. K. Gronley, and S. J. Mulroy, "Classification of walking handicap in the stroke population," Stroke, vol. 26, no. 6, pp. 982-989, 1995.

[41] J. Guo, J. Liu, C. Wang et al., "Differential involvement of rubral branches in chronic capsular and pontine stroke," NeuroImage: Clinical, vol. 24, p. 102090, 2019.

[42] M. Owen, C. Ingo, and J. P. A. Dewald, "Upper extremity motor impairments and microstructural changes in bulbospinal pathways in chronic hemiparetic stroke," Frontiers in Neurology, vol. 8, p. 257, 2017.

[43] G. A. Basile, M. Quartu, S. Bertino et al., "Red nucleus structure and function: from anatomy to clinical neurosciences," Brain Structure \& Function, vol. 226, no. 1, pp. 69-91, 2021.

[44] J. C. Stewart, M. O'Donnell, K. Handlery, and C. J. Winstein, "Skilled reach performance correlates with corpus callosum structural integrity in individuals with mild motor impairment after stroke: a preliminary investigation," Neurorehabilitation and Neural Repair, vol. 31, no. 7, pp. 657-665, 2017.

[45] J. Soulard, C. Huber, S. Baillieul et al., "Motor tract integrity predicts walking recovery: a diffusion MRI study in subacute stroke," Neurology, vol. 94, no. 6, pp. e583-e593, 2020.

[46] R. Schulz, H. Braass, G. Liuzzi et al., "White matter integrity of premotor-motor connections is associated with motor output in chronic stroke patients," NeuroImage: Clinical, vol. 7, pp. 82-86, 2015.

[47] J. D. Schaechter, Z. P. Fricker, K. L. Perdue et al., "Microstructural status of ipsilesional and contralesional corticospinal tract correlates with motor skill in chronic stroke patients," Human Brain Mapping, vol. 30, no. 11, pp. 3461-3474, 2009.

[48] S. H. Jang, "A review of the ipsilateral motor pathway as a recovery mechanism in patients with stroke," NeuroRehabilitation, vol. 24, no. 4, pp. 315-320, 2009.

[49] T. Sinkjaer, L. Miller, T. Andersen, and J. C. Houk, "Synaptic linkages between red nucleus cells and limb muscles during a multi-joint motor task," Experimental Brain Research, vol. 102, no. 3, pp. 546-550, 1995.

[50] A. Belhaj-Saif and P. D. Cheney, "Plasticity in the distribution of the red nucleus output to forearm muscles after unilateral lesions of the pyramidal tract," Journal of Neurophysiology, vol. 83, no. 5, pp. 3147-3153, 2000.

[51] H. J. ten Donkelaar, "Evolution of the red nucleus and rubrospinal tract," Behavioural Brain Research, vol. 28, no. 12, pp. 9-20, 1988.

[52] T. P. Hicks and S. Onodera, "The mammalian red nucleus and its role in motor systems, including the emergence of bipedalism and language," Progress in Neurobiology, vol. 96, no. 2, pp. 165-175, 2012.

[53] H. Barbeau and S. Rossignol, "Recovery of locomotion after chronic spinalization in the adult cat," Brain Research, vol. 412, no. 1, pp. 84-95, 1987.

[54] S. H. Jang, "The role of the corticospinal tract in motor recovery in patients with a stroke: a review," NeuroRehabilitation, vol. 24, no. 3, pp. 285-290, 2009.

[55] S. H. Jang, K. Kim, S. H. Kim, S. M. Son, W. H. Jang, and H. G. Kwon, "The relation between motor function of stroke patients and diffusion tensor imaging findings for the corticospinal tract," Neuroscience Letters, vol. 572, pp. 1-6, 2014. 
[56] Y. J. Yoo, J. W. Kim, J. S. Kim, B. Y. Hong, K. B. Lee, and S. H. Lim, "Corticospinal tract integrity and long-term hand function prognosis in patients with stroke," Frontiers in Neurology, vol. 10, p. 374, 2019.

[57] Q. Lu, G. Huang, L. Chen, W. Li, and Z. Liang, "Structural and functional reorganization following unilateral internal capsule infarction contribute to neurological function recovery," Neuroradiology, vol. 61, no. 10, pp. 1181-1190, 2019.

[58] S. Mohapatra, R. Harrington, E. Chan, A. W. Dromerick, E. Y. Breceda, and M. Harris-Love, "Role of contralesional hemisphere in paretic arm reaching in patients with severe arm paresis due to stroke: a preliminary report," Neuroscience Letters, vol. 617, pp. 52-58, 2016.

[59] J. C. Stewart, P. Dewanjee, G. Tran et al., "Role of corpus callosum integrity in arm function differs based on motor severity after stroke," NeuroImage: Clinical, vol. 14, pp. 641-647, 2017.

[60] Y. Peng, J. Liu, M. Hua, M. Liang, and C. Yu, "Enhanced effective connectivity from ipsilesional to contralesional M1 in well-recovered subcortical stroke patients," Frontiers in Neurology, vol. 10, p. 909, 2019.

[61] S. H. Jang, "A review of motor recovery mechanisms in patients with stroke," NeuroRehabilitation, vol. 22, no. 4, pp. 253-259, 2007.

[62] O. G. Filatova, L. J. van Vliet, A. C. Schouten, G. Kwakkel, F. C. T. van der Helm, and F. M. Vos, "Comparison of multi-tensor diffusion models' performance for white matter integrity estimation in chronic stroke," Frontiers in Neuroscience, vol. 12, p. $247,2018$.

[63] A. L. Borstad, S. Choi, P. Schmalbrock, and D. S. Nichols-Larsen, "Frontoparietal white matter integrity predicts haptic performance in chronic stroke," NeuroImage: Clinical, vol. 10, pp. 129-139, 2016.

[64] F. Song, F. Zhang, D. Z. Yin et al., "Diffusion tensor imaging for predicting hand motor outcome in chronic stroke patients," The Journal of International Medical Research, vol. 40, no. 1, pp. 126-133, 2012.

[65] S. H. Jang, S. M. Son, D. Y. Lee, and J. H. Hong, "Relationship between somatosensory function and the spinothalamocortical pathway in chronic stroke patients," Somatosensory \& Motor Research, vol. 30, no. 4, pp. 197-200, 2013.

[66] P. L. Khong, L. J. Zhou, G. C. Ooi, B. H. Chung, R. T. Cheung, and V.C. Wong, "The evaluation of Wallerian degeneration in chronic paediatric middle cerebral artery infarction using diffusion tensor MR imaging," Cerebrovascular Diseases, vol. 18, no. 3, pp. 240-247, 2004.

[67] X. Zhang, Y. Yan, F. Tong et al., "Progressive assessment of ischemic injury to white matter using diffusion tensor imaging: a preliminary study of a macaque model of stroke," Open Neuroimaging Journal, vol. 12, no. 1, pp. 30-41, 2018.

[68] R. A. Weber, E. S. Hui, J. H. Jensen et al., "Diffusional kurtosis and diffusion tensor imaging reveal different time-sensitive stroke-induced microstructural changes," Stroke, vol. 46, no. 2, pp. 545-550, 2015.

[69] J. C. Stewart, X. Tran, and S. C. Cramer, “Age-related variability in performance of a motor action selection task is related to differences in brain function and structure among older adults," NeuroImage, vol. 86, pp. 326-334, 2014.

[70] K. Y. Haaland, C. L. Elsinger, A. R. Mayer, S. Durgerian, and S. M. Rao, "Motor sequence complexity and performing hand produce differential patterns of hemispheric lateralization," Journal of Cognitive Neuroscience, vol. 16, no. 4, pp. 621-636, 2004.
[71] S. van Hees, K. McMahon, A. Angwin, G. de Zubicaray, S. Read, and D. A. Copland, "Changes in white matter connectivity following therapy for anomia post stroke," Neurorehabilitation and Neural Repair, vol. 28, no. 4, pp. 325-334, 2014.

[72] X. Zheng and G. Schlaug, "Structural white matter changes in descending motor tracts correlate with improvements in motor impairment after undergoing a treatment course of tDCS and physical therapy," Frontiers in Human Neuroscience, vol. 9, p. 229, 2015.

[73] D. S. Tuch, T. G. Reese, M. R. Wiegell, N. Makris, J. W. Belliveau, and V. J. Wedeen, "High angular resolution diffusion imaging reveals intravoxel white matter fiber heterogeneity," Magnetic Resonance in Medicine, vol. 48, no. 4, pp. 577-582, 2002.

[74] P. Vargas, M. Gaudron, R. Valabregue et al., "Assessment of corticospinal tract (CST) damage in acute stroke patients: comparison of tract-specific analysis versus segmentation of a CST template," Journal of Magnetic Resonance Imaging, vol. 37, no. 4, pp. 836-845, 2013. 